RECEIVED

HAY 064999

OSTI

DOE/SF/194:60--299-Pt.2
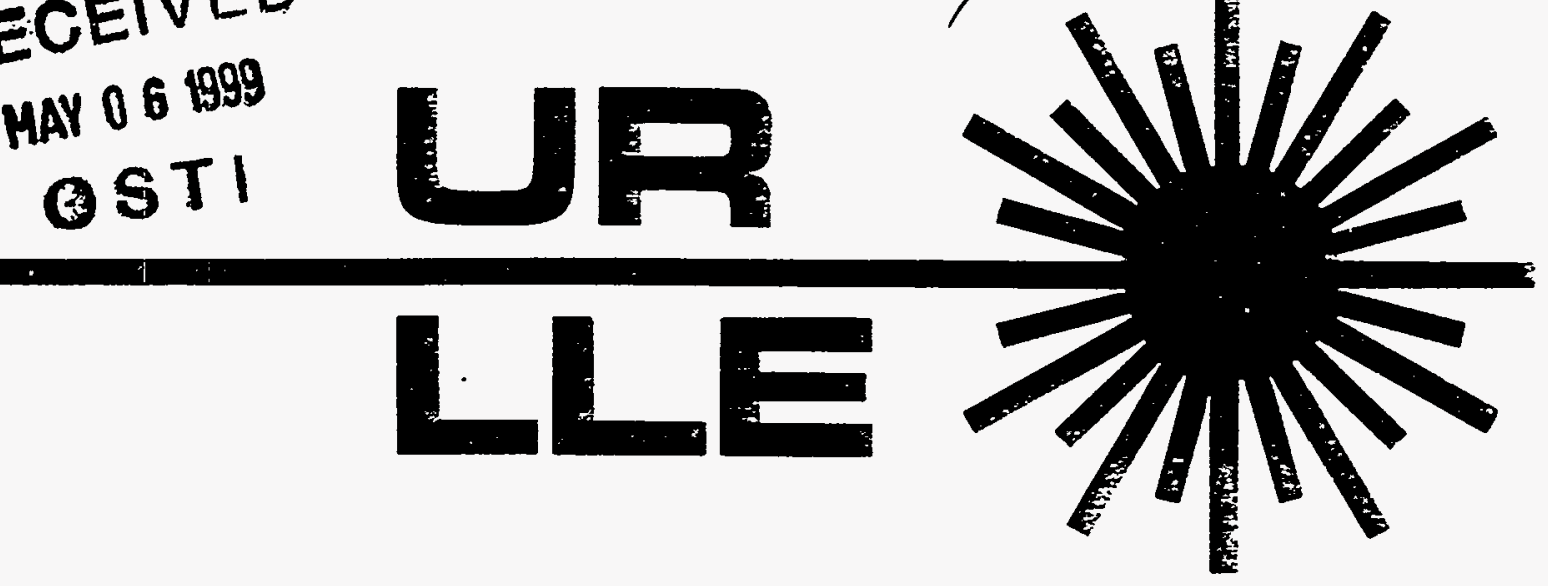

1998 SUMMER RESEARCH PROGRAM FOR HIGH SCHOOL JUNIORS

AT THE

UNIVERSITY OF ROCHESTER'S

LABORATORY FOR LASER ENERGETICS

STUDENT RESEARCH REPORTS

PROJECT COORDINATOR

Dr. R. Stephen Craxton

March 1999

OISTAIBUTION OF THIS DOCUMEAT IS UHATER

Laboratory Report 300

MASTER

Laboratory for Laser Energetics University of Rocnester 


\section{DISCLAIMER}

This report was prepared as an account of work sponsored by an agency of the United States Government. Neither the United States Government nor any agency thereof, nor any of their employees, makes any warranty, express or implied, or assumes any legal liability or responsibility for the accuracy, completeness, or usefulness of any information, apparatus, product, or process disclosed, or represents that its use would not infringe privately owned rights. Reference herein to any specific commercial product, process, or service by trade name, trademark, manufacturer, or otherwise does not necessarily constitute or imply its endorsement, recommendation, or favoring by the United States Government or any agency thereof. The views and opinions of authors expressed herein do not necessarily state or reflect those of the United States Government or any agency thereof. 


\section{DISCLAIMER}

Portions of this document may be illegible in electronic image products. Images are produced from the best available original document. 


\title{
1998 SUMMER RESEARCH PROGRAM FOR HIGH SCHOOL JUNIORS \\ AT THE \\ UNIVERSITY OF ROCHESTER'S \\ LABORATORY FOR LASER ENERGETICS
}

\author{
STUDENT RESEARCH REPORTS
}

\section{PROGRAM COORDINATOR}

\author{
Dr. R. Stephen Craxton \\ LABORATORY FOR LASER ENERGETICS \\ University of Rochester \\ 250 East River Road \\ Rochester, NY 14623-1299
}

During the summer of 1998,11 students from Rochester-area high schools participated in the Laboratory for Laser Energetics' Summer High School Research Program. The goal of this program is to excite a group of high school students about careers in the areas of science and technology by exposing them to research in a state-ofthe-art environment. Too often, students are exposed to "research" only through classroom laboratories that have prescribed procedures and predictable results. In LLE's summer program, the students experience all of the trials, tribulations, and rewards of scientific research. By participating in research in a real environment, the students often 
become more excited about careers in science and technology. In addition, LLE gains from the contributions of the many highly talented students who are attracted to the program.

The students spent most of their time working on their individual research projects with members of LLE's technical staff. The projects were related to current research activities at LLE and covered a broad range of areas of interest including optics, spectroscopy, chemistry, diagnostic development, and materials science. The students, their high schools, their LLE supervisors and their project titles are listed in the table. Their written reports are collected in this volume.

The students attended weekly seminars on technical topics associated with LLE's research. Topics this year included lasers, fusion, holography, nonlinear optics, global warming, and scientific ethics. The students also received safety training, learned how to give scientific presentations, and were introduced to LLE's resources, especially the computational facilities.

The program culminated with the High School Student Summer Research Symposium on 26 August at which the students presented the results of their research to an audience that included parents, teachers, and members of LLE. Each student spoke for approximately ten minutes and answered questions. At the symposium an Inspirational Science Teacher award was presented to Mr. David Crane, a chemistry teacher at Greece Arcadia High School. This annual award honors a teacher, nominated by alumni of the LLE program, who has inspired outstanding students in the areas of science, mathematics, and technology. 


\begin{tabular}{|c|c|c|c|}
\hline \multicolumn{4}{|c|}{ High School Students and Their Projects (1998) } \\
\hline Student & High School & Supervisor & Project \\
\hline Steven Corsello & Pittsford Mendon & K. Marshall & $\begin{array}{l}\text { Computer-Aided Design and } \\
\text { Modeling of Nickel Dithiolene } \\
\text { Near-Infrared Dyes }\end{array}$ \\
\hline Peter Grossman & Wilson Magnet & R. S. Craxton & $\begin{array}{l}\text { Group Velocity Effects in } \\
\text { Broadband Frequency } \\
\text { Conversion on OMEGA }\end{array}$ \\
\hline Joshua Hubregsen & Pittsford Sutherland & S. Jacobs & $\begin{array}{l}\text { A Study of Material Removal } \\
\text { During Magnetorheological } \\
\text { Finishing (MRF) }\end{array}$ \\
\hline Nieraj Jain & Pittsford Sutherland & M. Guardelben & $\begin{array}{l}\text { Analyzing Algorithms for } \\
\text { Nonlinear and Spatially } \\
\text { Nonuniform Phase Shifts in } \\
\text { the Liquid Crystal Point } \\
\text { Diffraction Interferometer }\end{array}$ \\
\hline Leslie Lai & Pittsford Mendon & M. Wittman & $\begin{array}{l}\text { The Use of Design-of- } \\
\text { Experiments Methodology to } \\
\text { Optimize Polymer Capsule } \\
\text { Fabrication } \\
\end{array}$ \\
\hline Irene Lippa & Byron-Bergen & K. Marshall & $\begin{array}{l}\text { Synthesis and Analysis of } \\
\text { Nickel Dithiolene Dyes in a } \\
\text { Nematic Liquid Crystal Host }\end{array}$ \\
\hline $\begin{array}{l}\text { Phillip } \\
\text { Ostromogolsky }\end{array}$ & Brighton & F. Marshall & $\begin{array}{l}\text { Investigation of the X-Ray } \\
\text { Diffraction Properties of a } \\
\text { Synthetic Multilayer }\end{array}$ \\
\hline $\begin{array}{l}\text { Michael } \\
\text { Schubmehl }\end{array}$ & The Harley School & R. Epstein & $\begin{array}{l}\text { An Analysis of the Uncertainty } \\
\text { in Temperature and Density } \\
\text { Estimates from Fitting Model } \\
\text { Spectra to Data }\end{array}$ \\
\hline Joshua Silbermann & Penfield & P. Jannimagi & $\begin{array}{l}\text { Automated CCD Camera } \\
\text { Characterization }\end{array}$ \\
\hline Abigail Stern & The Harley School & J. Knauer & $\begin{array}{l}\text { Design and Testing of a } \\
\text { Compact X-Ray Diode }\end{array}$ \\
\hline Amy Turner & Churchville-Chili & R. S. Craxton & $\begin{array}{l}\text { Ray Tracing Through the Liquid } \\
\text { Crystal Point Diffraction } \\
\text { Interferometer }\end{array}$ \\
\hline
\end{tabular}


A total of 91 high school students have participated in the program since it began in 1989. The students this year were selected from approximately 60 applicants. Each applicant submitted an essay describing their interests in science, a copy of their transcript, and a letter of recommendation from a science or math teacher.

LLE plans to continue this program in future years. The program is strictly for students from Rochester-area high schools who have just completed their junior year. Applications are generally mailed out in February with an application deadline near the end of March. For more information about the program or an application form, please contact Dr. R. Stephen Craxton at LLE.

This program was supported by the U.S. Department of Energy Office of Inertial Confinement Fusion under Cooperative Agreement No. DE-FC03-92SF19460. 


\section{GROUP VELOCITY EFFECTS IN BROADBAND \\ FREQUENCY CONVERSION ON OMEGA}

by

\section{PETER GROSSMAN}

Wilson Magnet High School

Rochester, NY

W 


\title{
Group Velocity Effects in Broadband Frequency Conversion on OMEGA
}

\author{
Peter M. Grossman
}

\author{
Advisor: R. S. Craxton
}

\section{Laboratory for Laser Energetics University of Rochester}

\section{Summer High School Research Program 1998}

\section{Introduction:}

Currently, the world relies mainly on fossil fuels as an energy source. There are several problems with this source of energy, mainly that fossil fuels are a limited resource, and that the burning of fossil fuels releases many pollutants into the atmosphere. Therefore, research is being performed on different energy sources which would be either renewable or unlimited, and which have little or no negative impact on the environment.

One possible energy source which meets these requirements is nuclear fusion. At the University of Rochester Laboratory for Laser Energetics (LLE), experiments are being carried out on a method of fusing ions that is called Inertial Confinement Fusion (ICF). ICF at LLE is performed by compressing a small spherical fuel pellet of one millimeter diameter using a powerful laser called OMEGA. The pellet has an outer shell comprised of either glass or plastic, and is filled with a gaseous mixture of deuterium and tritium. As the sixty beams from OMEGA strike the fuel pellet, the outer 
layer becomes a plasma and ablates away from the pellet at speeds of approximately 1,000 kilometers per second [1]. The ablating plasma essentially acts as a rocket, and due to Newton's third law, the inner material accelerates toward the center of the pellet. It is desired to compress the fuel pellet as much as 50 times. This compression ratio is enough to cause a large number of fusion reactions to occur between the deuterium and tritium ions in the core of the fuel pellet [1]. These reactions produce alpha particles (helium nuclei) and energetic neutrons.

The powerful lasers needed for ICF can only produce light in the infrared wavelengths. However, the one micron wavelength produced by the neodymium glass that powers OMEGA and other lasers used for fusion research does not efficiently compress the fuel pellet. This happens because the infrared light is not well absorbed by the target, and because of the creation of suprathermal electrons. These suprathermal electrons preheat the fuel, adding extra resistance to compression [1].

To eliminate these problems associated with longer wavelengths of light, the process of frequency converting the laser beam was invented. This process efficiently converts the initial beam to a beam which has three times the frequency and one third the wavelength. The third-harmonic beam, in the UV range, has a better absorption rate [1].

\section{Frequency Conversion:}

The frequency conversion process relies on potassium dihydrogen phosphate (KDP) crystals [1]. These crystals can be used to generate different harmonics of a certain input wave, depending on how they are arranged, how many crystals the wave passes through, and how the crystals are oriented. The crystals take laser pulses and split them into separate components polarized parallel to the two axes, the extraordinary (e) axis, and the ordinary (0) axis [2]. In the crystals, the component of the pulse that is polarized parallel to the e axis moves faster than the portion of the wave parallel to the 0 
layer becomes a plasma and ablates away from the pellet at speeds of approximately 1,000 kilometers per second [1]. The ablating plasma essentially acts as a rocket, and due to Newton's third law, the desired inner material accelerates toward the center of the pellet. It is designed to compress the fuel pellet as much as 50 times. This compression ratio is enough to cause a large number of fusion reactions to occur between the deuterium and tritium ions in the core of the fuel pellet [1]. These reactions produce alpha particles (helium nuclei) and energetic neutrons.

The powerful lasers needed for ICF can only produce light in the infrared wavelengths. However, the one micron wavelength produced by the neodymium glass that powers OMEGA and other lasers used for fusion research does not efficiently compress the fuel pellet. This happens because the infrared light is not well absorbed by the target, and because of the creation of suprathermal electrons. These suprathermal electrons preheat the fuel, adding extra resistance to compression [1].

To eliminate these problems associated with longer wavelengths of light, the process of frequency converting the laser beam was invented. This process efficiently converts the initial beam to a beam which has three times the frequency and one third the wavelength. The third-harmonic beam, in the UV range, has a better absorption rate [1].

\section{Frequency Conversion:}

The frequency conversion process relies on potassium dihydrogen phosphate (KDP) crystals [1]. These crystals can be used to generate different harmonics of a certain input wave, depending on how they are arranged, how many crystals the wave passes through, and how the crystals are oriented. The crystals take laser pulses and split them into separate components polarized parallel to the two axes, the extraordinary (e) axis, and the ordinary (o) axis [2]. In the crystals, the component of the pulse that is polarized parallel to the $\mathrm{e}$ axis moves faster than the portion of the wave parallel to the 0 
axis. This difference can cause the different parts of the input pulse to separate from each other. Since the pulses need to remain intact as much as possible to have high conversion efficiency, this effect can lower the conversion efficiency. Normally this separation is only a factor for short pulses, but it can also have an impact when dealing with a small part of a longer pulse.

The current setup on the OMEGA laser system uses two crystals, and a polarizer that polarizes the light at $35^{\circ}$. This setup is used to generate the third harmonic of the input laser beam. Though this setup is highly efficient, generating the third harmonic at an $80 \%$ efficiency, it only does so for a narrow range of wavelengths. The first step in the process is to polarize the light so that one IR photon is traveling polarized parallel to the e axis and two photons are polarized parallel to the o axis. The first (doubler) crystal these photons interact with causes two photons to combine to form one green photon polarized parallel to the e axis of the first crystal, and o axis of the tripler crystal. The next (tripler) crystal that the photons pass though combines the green light produced in the doubler crystal to mix with the leftover IR photons. A tuned crystal, meaning untilted, will efficiently convert the normal input wavelength. However, the crystal may be very slightly tilted intentionally, or detuned, to efficiently convert a different wavelength.

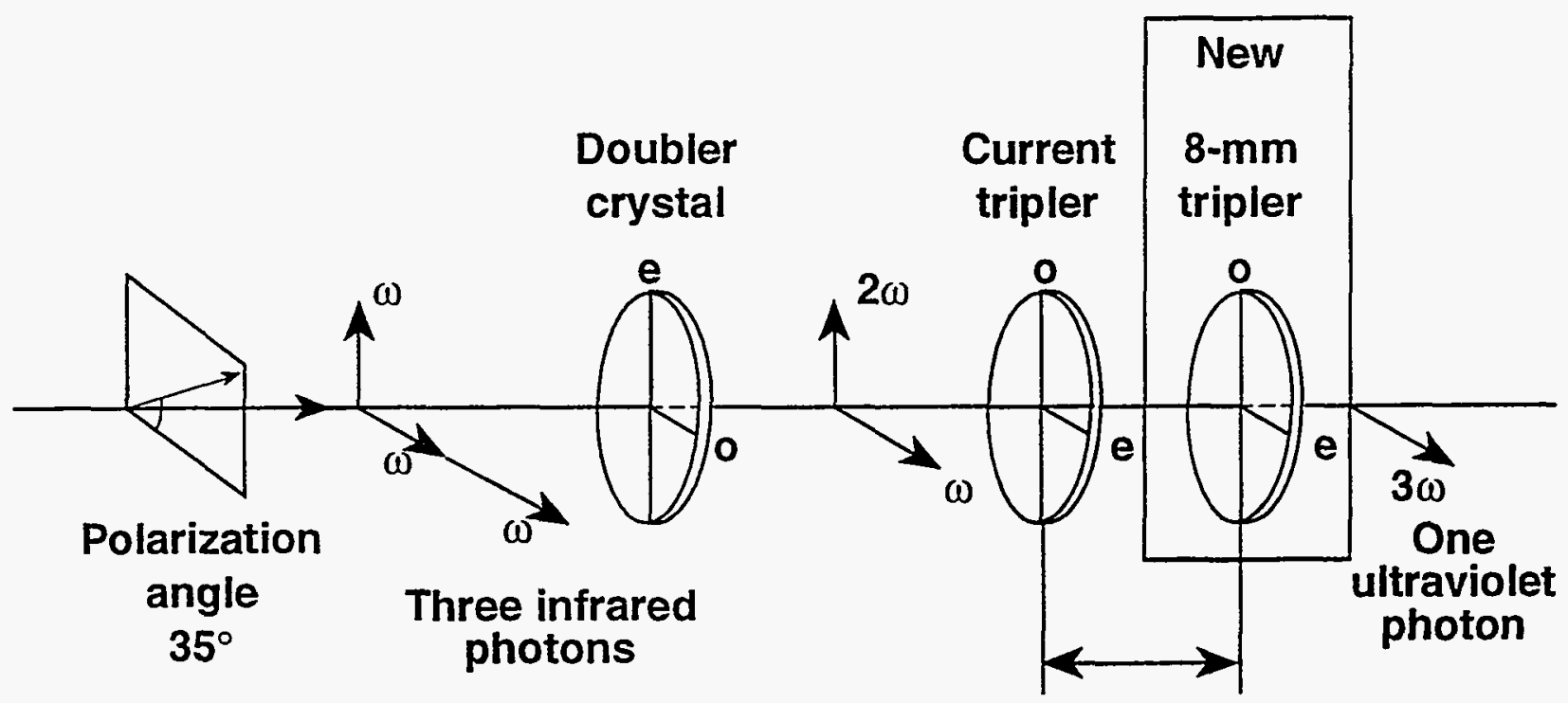

Fig. 1 Diagram of the new frequency conversion scheme to be placed on OMEGA. 
In order to increase the range of wavelengths where the conversion efficiency is high, a second tripler crystal is being added to each beam of the OMEGA laser system (see Fig. 1, above). The ability to detune each crystal used in the tripling process to convert a different wavelength allows the bandwidth to be increased [3]. Based on Oskoui's predictions [4], this addition of a second tripler crystal will increase the bandwidth of efficient third harmonic generation (THG) conversion by approximately 3 times (see Fig. 2. below). This effect has been experimentally demonstrated, and results are in good agreement with the computer model [5].

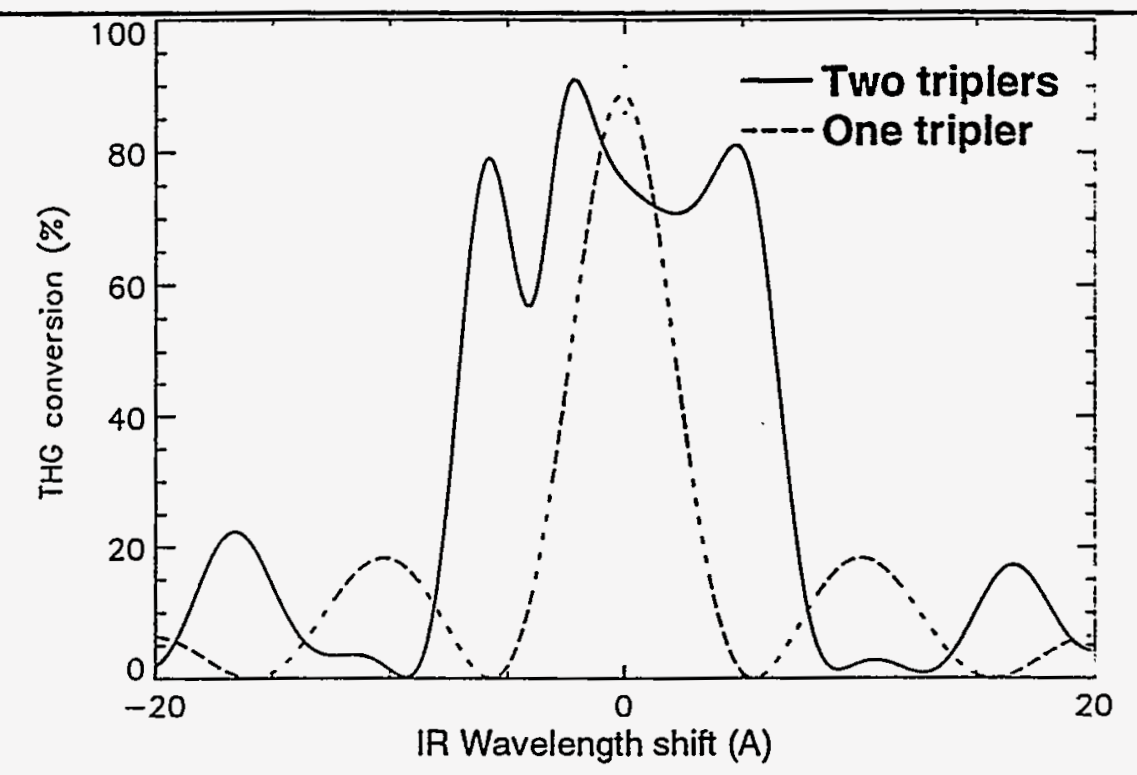

Fig. 2 THG conversion versus wavelength shift, with both the old and future OMEGA setups.

\section{Smoothing by Spectral Dispersion:}

The bandwidth at which the crystals efficiently convert the input laser beam to the third harmonic is important because a larger bandwidth allows for a smoother laser. The smoothing of the laser beam is accomplished by a process called Smoothing by Spectral Dispersion (SSD)[6]. Due to aberrations throughout each beamline, the laser beam has many hot and cold spots across its diameter by the time that the beam is focused down to the target. The laser beams which strike the target do not uniformly irradiate the target, due to the fact that they are not smooth. This leads to higher nonuniformity, which hurts the fuel's ability to fuse, since the compression is not even in all 
directions.

SSD starts when the laser pulse is diffracted by a specially made grating. The pulse then travels through a device called an electro-optic phase modulator. This device imparts a slightly different wavelength to different parts of the beam, cycling through a slight red-shift and slight blue-shift very rapidly with a modulation frequency that is typically $10 \mathrm{GHz}$. The beam is then diffracted by a second grating which makes the beam travel parallel to its original course. As the beam nears the target chamber after frequency tripling, it encounters what is called a phase plate. This phase plate breaks the beam up into many different smaller beamlets, which interfere with one another. Due to the differing wavelengths across the diameter of the beam at any given time, different beamlets are diffracted onto a different place on the target. The rapid oscillation in beam pointing produces a smoother shot on target from each beamline over time.
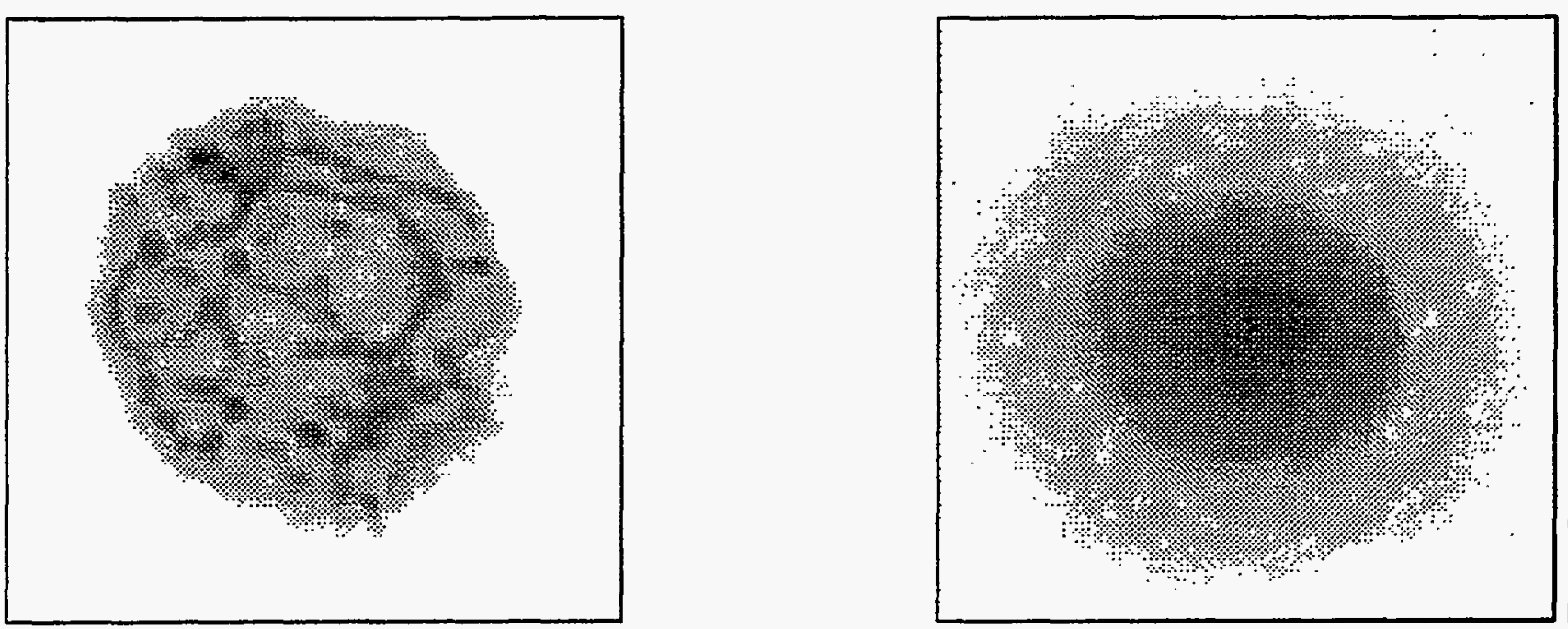

Fig. 3 Picture of unsmoothed (left) and smoothed (right) laser spot on target.

The objective of my project was to model the frequency converted SSD beam on the upgraded version of OMEGA with the additional tripling crystal, and to find out what happens to conversion efficiency as modulation frequency is increased, when it is expected that the differential velocities of parts of the input pulse mentioned earlier will have an effect. Using the PV-WAVE programming language, this situation has been modeled. In the future, SSD designs may call for higher modulation 
frequencies, and it is important to know the effect of these higher frequencies on conversion efficiency.

\section{Description of the Code:}

The conversion efficiency of the crystals can be calculated by using the frequency conversion equations (below) $[2,7]$.

$$
\begin{aligned}
& \frac{\delta E_{1}}{\delta Z}+\left(\frac{1}{V_{1}}-\frac{1}{V_{\text {ref }}}\right) \frac{\delta E_{1}}{\delta T}=-i \cdot K_{1} \cdot E_{3} \cdot E_{2}^{*} \cdot \exp (-i \cdot \Delta k \cdot Z) \\
& \frac{\delta E_{2}}{\delta Z}+\left(\frac{1}{V_{2}}-\frac{1}{V_{\text {ref }}}\right) \frac{\delta E_{2}}{\delta T}=-i \cdot K_{2} \cdot E_{3} \cdot E_{1}^{*} \cdot \exp (-i \cdot \Delta k \cdot Z) \\
& \frac{\delta E_{3}}{\delta Z}+\left(\frac{1}{V_{3}}-\frac{1}{V_{\text {ref }}}\right) \frac{\delta E_{3}}{\delta T}=-i \cdot K_{3} \cdot E_{1}: E_{2} \cdot \exp (i \cdot \Delta k \cdot Z)
\end{aligned}
$$

The electric field, which relates to the intensity of the wave, can be represented by a complex number which gives the field's amplitude and phase. The equations used are all interrelated. When in the first crystal, $E_{1}$ and $E_{2}$ describe the rate of growth of the 0 and e polarized waves of the fundamental harmonic, respectively. Both of these waves are the "red" waves, which means they are in the IR. The "green" wave of the second harmonic is represented by $E_{3}$ in the first crystal, and by $E_{2}$ in both tripling crystals. In both of the tripling crystals, the $E_{1}$ "red" waves and the $E_{2}$ "green" waves combine to form the $E_{3}$ "blue" UV waves, the end result of the conversion process and it is the UV beam which illuminates the target.

The basic equations used in the code involve 4 key terms. The first term, $\delta E / \delta Z$, refers to the rate of change of the electric field as the puise passes through the crystal. The next term, $1 / \mathrm{V} \bullet \delta \mathrm{E} / \delta \mathrm{T}$, is the group velocity term. This term deals with the speed at which the pulse travels through the crystal relative to some reference frame moving with the speed $V_{\text {rer, }}$ an important factor when dealing with short pulses. The main term, $-\mathrm{iK}_{3} \mathrm{E}_{1} \mathrm{E}_{2}$, describes the conversion of $E_{1}$ and $E_{2}$ to $E_{3}$. The last term, $(\mathrm{i} \bullet \Delta \mathrm{K} \cdot \mathrm{z})$, is the phase difference, which comes into play when the crystals are not properly tuned and 
the different waves of the laser beam have a chance to get out of phase. This last term drops out when the crystals are tuned properly, because $\Delta \mathrm{K}$ turns out to be zero. When the crystals are not properly tuned and the waves get out of phase, the waves can sometimes cancel each other out, leading to lower conversion efficiencies. However, the crystals are intentionally detuned slightly with the new setup in OMEGA. This is the detuning which allows each crystal to efficiently convert a different wavelength. This is the trick used to obtain a broad bandwidth of efficient conversion [3].

The different group velocity terms can cause a walkoff between the different beam harmonics. The group velocity $V_{1}$ has a value of $2.0178 \times 10^{8}$ meters per second, $V_{2}$ is $1.9394 \times 10^{8}$ meters per second. and $V_{3}$ is $1.9216 \times 10^{8}$ meters per second in both of the tripler crystals. The transit time difference in the tripler crystals between the first and third harmonics is about 5 picoseconds, and about 1 picosecond between the second and third harmonics.

Another part of the code is the initial setup of the electric field (below).

$$
E(t)=E_{o} \cdot \exp \left[i \cdot \delta \cdot \sin \left(2 \pi \nu_{m}\left(t-t_{o}\right)\right)\right]
$$

This is an important part of the code, as it is the part which allows changes as to how SSD is implemented. The SSD has a modulation frequency $v_{m}$ and a modulation index $\delta$. These values multiplied are proportional to the full bandwidth $\Delta \lambda_{\text {full }}$ (below).

$$
\Delta \lambda_{f u l l}=4 \pi \nu_{m} \delta
$$

The time for each cycle through SSD is given by $1 / v_{\mathrm{m}}$. The initial electric amplitude field $\mathrm{E}_{0}$ corresponds to the input intensity of $1.5 \mathrm{GW} / \mathrm{cm}^{2}$.

The method used to calculate the final value of each electric field was the half-step/whole-step method of integration. This method works by calculating the derivative, or slope, of the function at a point, and using that value to calculate a value of the electric field midway between the first and second points of integration. A derivative is then calculated at the intermediate value, the half-step, and these values are used to find the value for the second point of integration. This process is then 


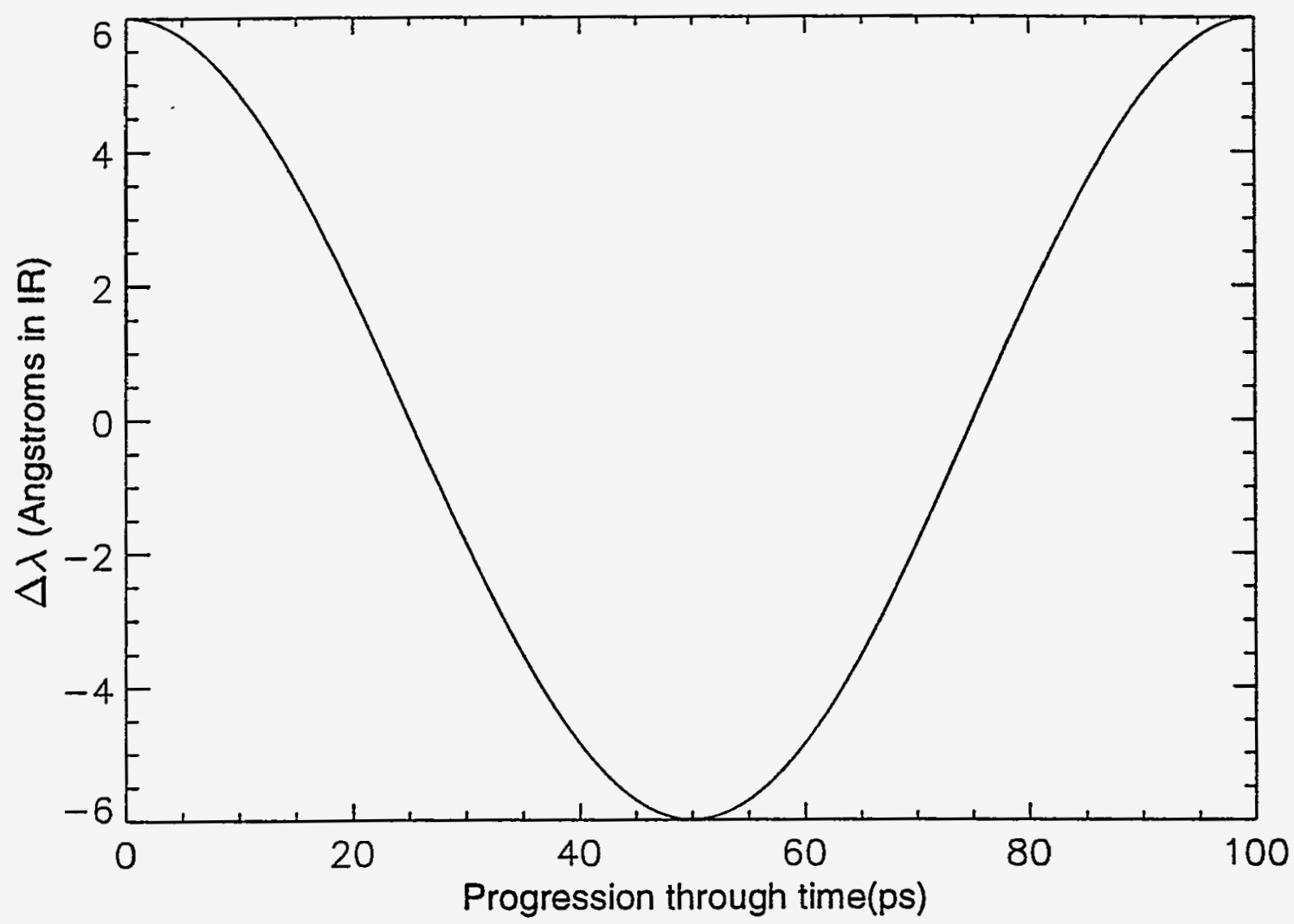

Fig. 4 Graph of $\Delta \lambda$ versus time through the cycle of SSD. Here $v_{m}=10 \mathrm{GHz}, \delta=16.2$, and $\Delta \lambda_{\text {full }}=12$ Angstroms.

used repeatedly to find a value for the electric field of all three waves at many different points through the crystal and at all possible times. This method of integration is useful because it is highly accurate, and does not require a large number of steps through either space or time.

The number of steps through time had to fall within a certain range, depending on the value of $\delta$, the modulation index, for a particular run. While the number of steps through space was held at 200 for all the runs, the number of steps through time was as high as 2000 for a period of 1000 picoseconds, and as low as 100 for periods of 10 picoseconds or less. It seems that the number of time steps relates to the interval being looked at in that the code requires about one-tenth to one-half picoseconds per time step. For modeling the setup to be used on OMEGA, 600 time steps were adequate. When too many time steps were used, the solution did not converge. If too few time steps were used the effect was less dramatic, but still caused an incorrect graph and improper value for conversion efficiency. 
My code was able to calculate the efficiency of third harmonic conversion through one cycle of SSD in two different ways. Method one was the correct way, which took into account the group velocity terms. This method used a single value for $\Delta \mathrm{K}$ throughout all times. Though there were different values of $\Delta \mathrm{K}$ for each crystal, all of the phase modulation was modeled by using a phase-modulated setup for the initial electric field.

The second method used an initial electric field that was constant in time, but a varying $\Delta \mathrm{K}$ value based on the $\Delta \lambda$ for that time (see Fig. 4 , above). This method did not take into account the group velocity terms, and therefore is less accurate.

\section{Results:}

All runs of the program used a full bandwidth $\Delta \lambda_{\text {full }}$ of 12 Angstroms in pump wavelength. This value is the design for the OMEGA system. The design also calls for a modulation frequency of $10 \mathrm{GHz}$, corresponding to an SSD cycle of 100 picoseconds. For this modulation frequency, the corresponding $\delta$ to make a bandwidth of 12 Angstroms was approximately 16.2. The result of these

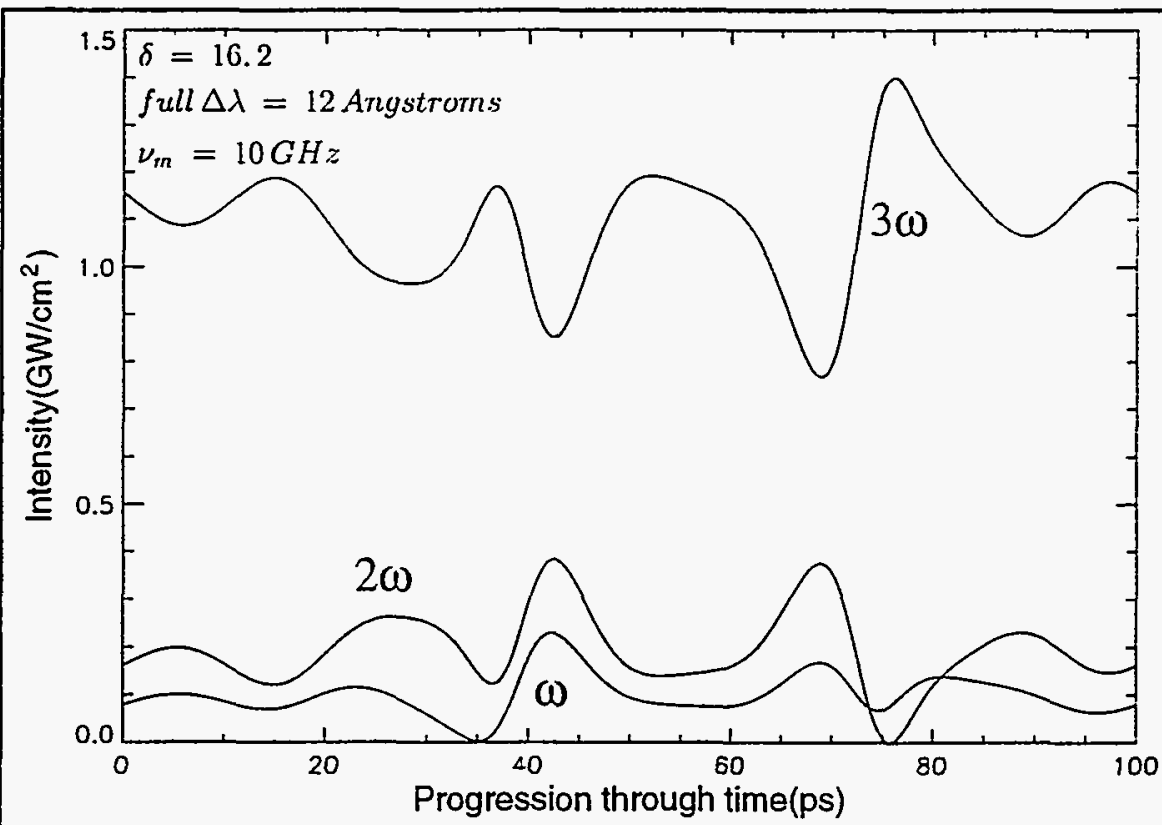

Fig. 5 Graph of intensity versus time at all three wavelengths for the proposed OMEGA design, using the correct method of calculation. The input beam had an intensity of $1.5 \mathrm{GW} / \mathrm{cm}^{2}$. parameters (see Fig. 5, left)

was a third harmonic

generation conversion

efficiency not far off from that

which was calculated using

the second method. At the

settings for OMEGA, the

calculated efficiency for both

methods was approximately

$73 \%$, averaged over the 100 


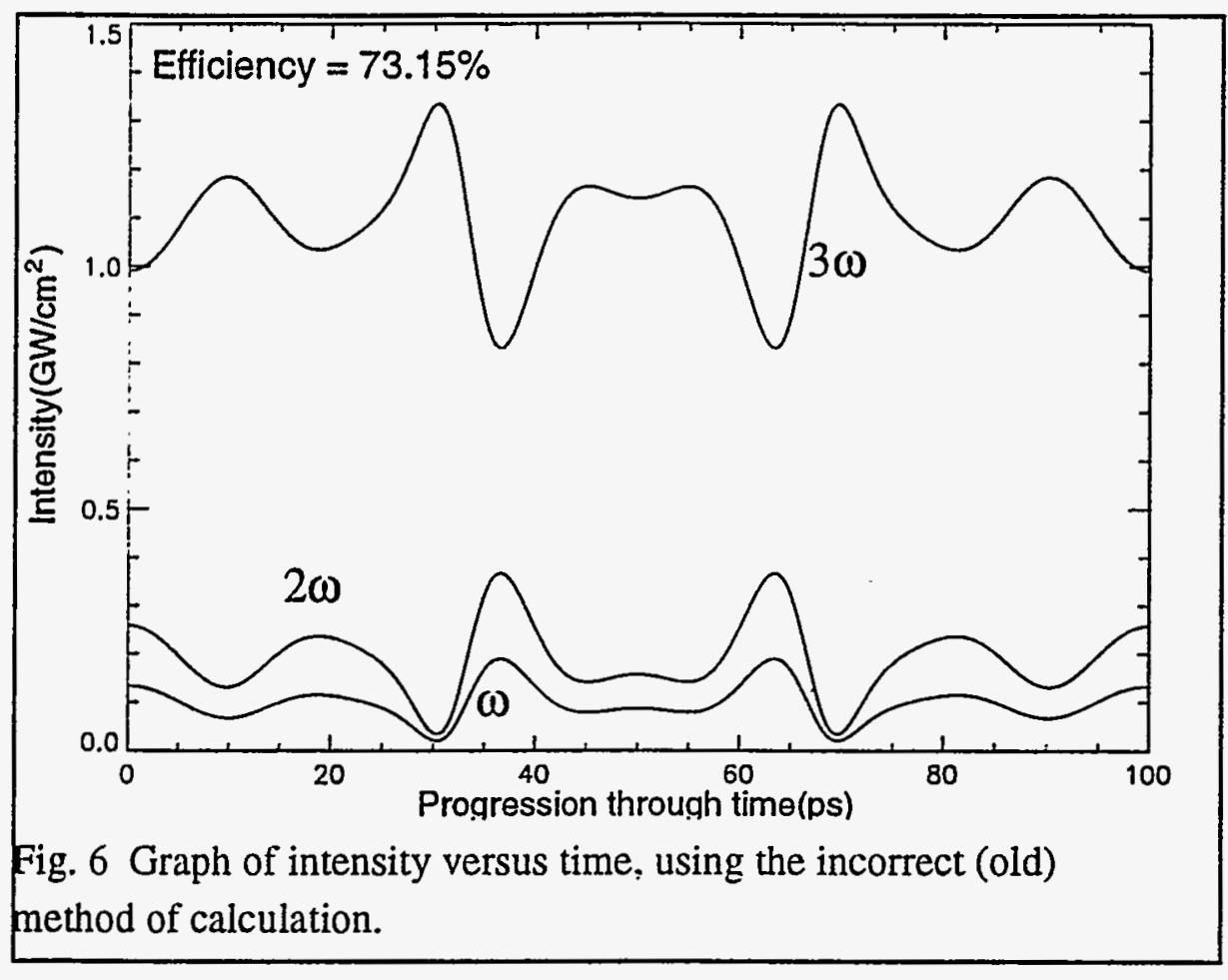

picosecond period.

Using the second

method of calculation,

the graph of intensity

versus time (see Fig. 6 ,

left) is completely

symmetrical. The $\Delta \lambda$

graph itself is symmetrical,

and the phase modulation

in the second method of

calculation was based essentially on that graph, without the effect of group velocities. The graph

produced by this second method is what would be the result of tracing back and forth through plus or minus 6 Angstroms along the solid graph shown above in Fig. 2. The group velocity has an effect of approximately two picoseconds per centimeter of crystal, so the group velocity should not have had a large impact.

While the conversion

efficiency varies between

approximately $60 \%$ and $90 \%$,

the average conversion

efficiency is $73 \%$. The

fluctuations seen in the third

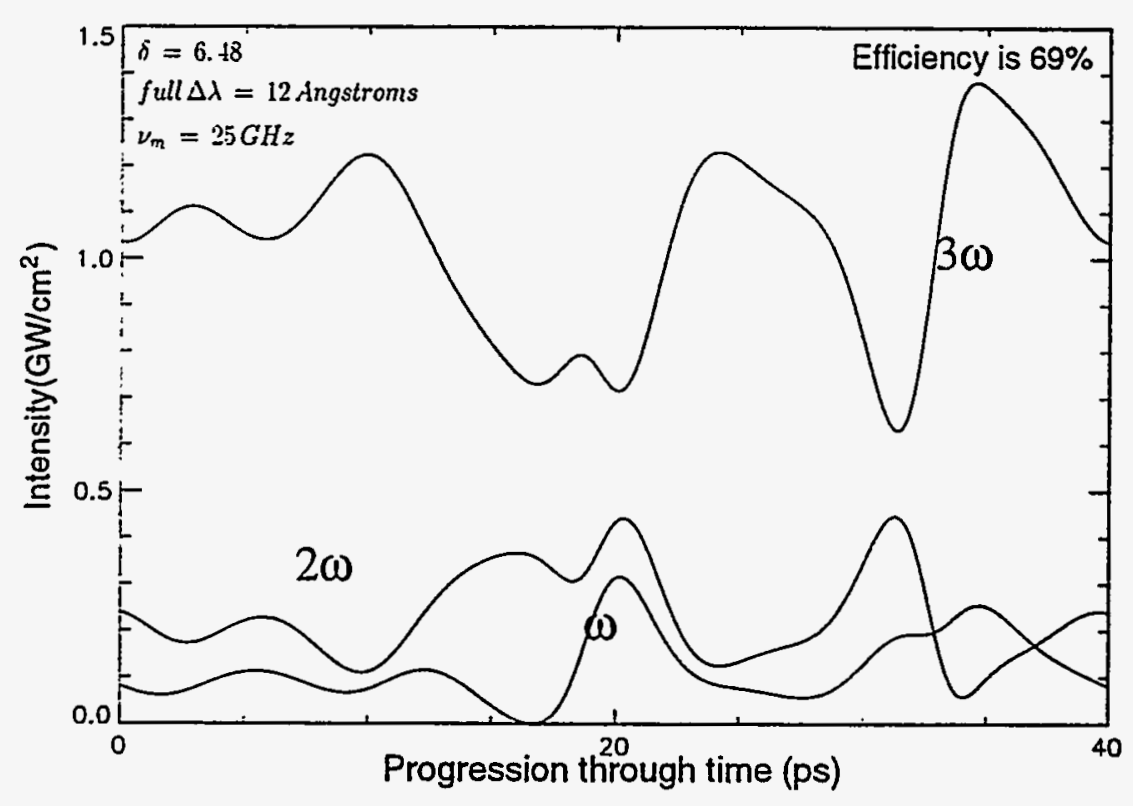

Fig. 7 Graph of intensity versus time, using the correct method of calculation. This graph is for a modulation frequency of $25 \mathrm{GHz}$, higher than the actual OMEGA setup.

harmonic in these graphs over 
the $100 \mathrm{ps}$ interval would never be seen by the target since, at any time, different parts across the diameter of the beam have different wavelength shifts.

Other values for modulation frequency were also tested for the same full bandwidth of 12 Angstroms. At shorter time intervals, the group velocity effects would be magnified. However, the drop-off in conversion is not large at values of modulation frequency close to $10 \mathrm{GHz}$. For example, the efficiency at $25 \mathrm{GHz}$ was $69 \%$ (see Fig. 7, above), only around $4 \%$ lower than the conversion efficiency for the $10 \mathrm{GHz}$ case. At this modulation frequency, the graph is even more asymmetric, far more complex than the $10 \mathrm{GHz}$ case. Since at higher modulation frequencies the time for one cycle through SSD is shorter, this result was expected.

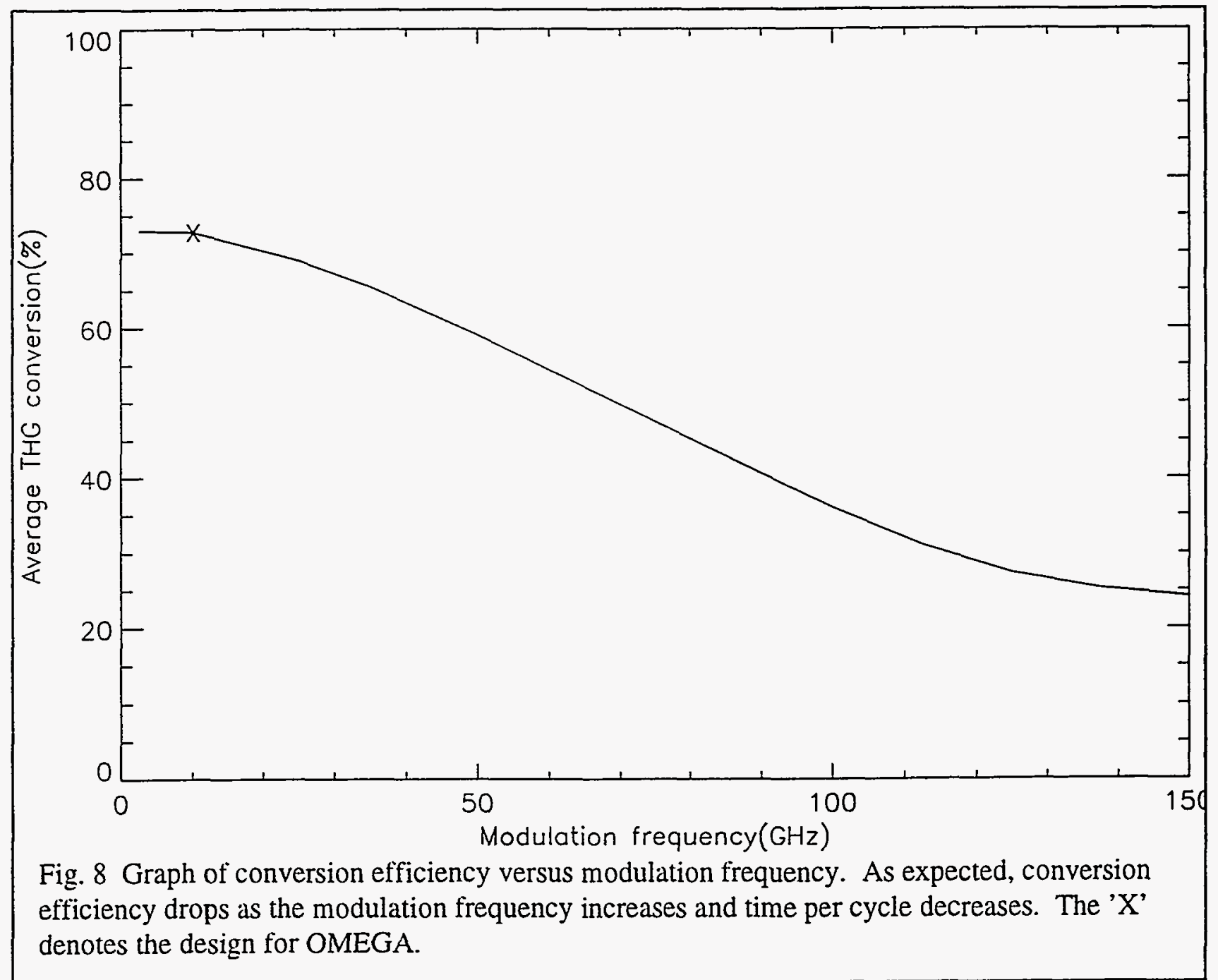


The results of many different runs allowed me to produce a graph of conversion efficiency versus modulation frequency (see Fig. 8 , above). The general downward trend of the graph is easily explainable as the increasing effect of group velocity as the time for each cycle grew progressively shorter. The graph seems to begin to level out at the end, near the $125 \mathrm{GHz}$ area. The graph shows that around the OMEGA design, the conversion efficiency is still quite high. Serious conversion loss does not occur until approximately $30 \mathrm{GHz}$, where the graph's downward slope increases. This result is also applicable to the National Ignition Facility (NIF). One proposal for the NIF called for a modulation frequency which averaged to $50 \mathrm{GHz}$ [8]. However, based on my program's calculations, this would result in a serious conversion loss, which would be possibly unacceptable to the NIF.

The code that was written was also used to model a different problem, that of very short laser pulses. These short pulses were modeled using the two tripler system specifications for the OMEGA laser system, but the way the initial electric field was input was completely different. The initial electric field was set as having a Gaussian shape in time and no phase modulation with the full width at half maximum (FWHM) set for one and two picoseconds on different runs of the program.
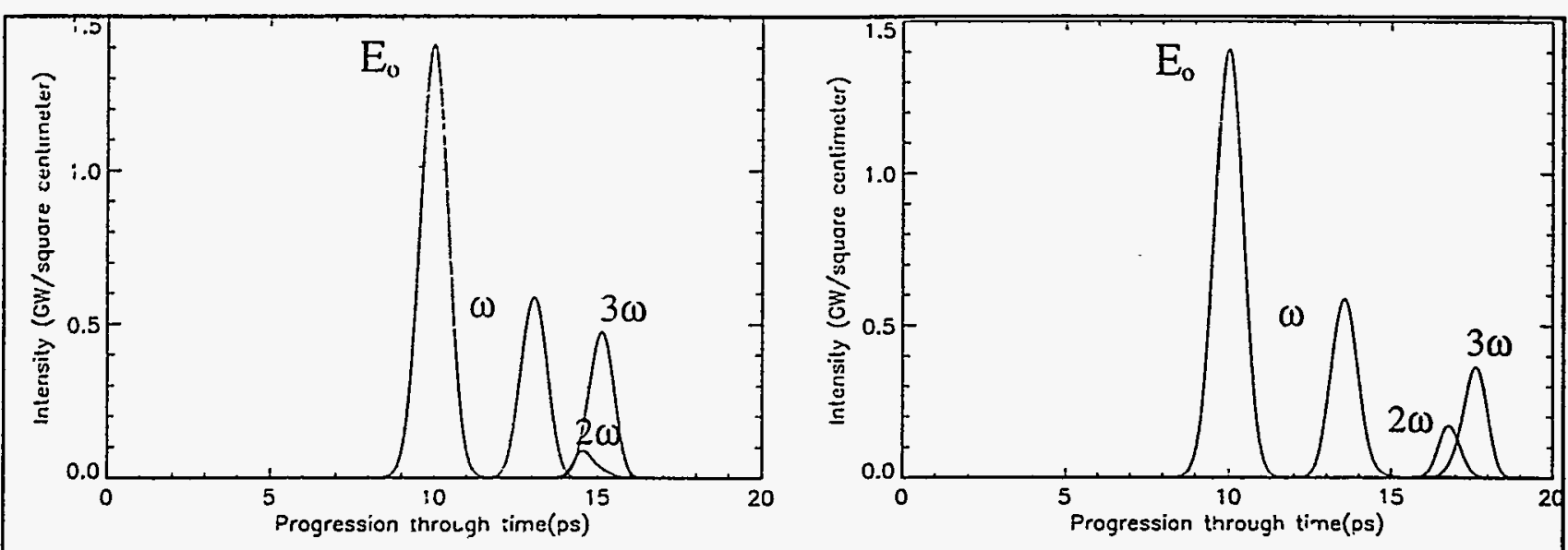

Fig. 9 Graphs of intensity versus time for short pulse with one picosecond full-width at half maximum. Left: one tripling crystal, right: two tripling crystals. Conversion efficiency was not helped by the addition of the second tripler.

It was found that the conversion efficiency for these short pulses was not improved using the two tripler system. With a FWHM of one picosecond, the conversion efficiency dropped by 
approximately $7 \%$, from $30 \%$ with one tripler to $23 \%$ when using the two tripler scheme (see Fig. 9 , above). With a FWHM of two picoseconds, the conversion efficiency was lowered by $22 \%$, dropping from $58 \%$ using one tripler to $36 \%$ using two triplers. For a one picosecond puise the corresponding bandwidth is 17 Angstroms, while for two picoseconds the corresponding bandwidth is 8.5

Angstroms. It appears that the dual-tripler scheme helps phase-modulated but not amplitude-modulated bandwidth. It is fortunate that the only type of bandwidth of interest to OMEGA is phase-modulated bandwidth.

\section{Conclusion:}

The PV-WAVE computer program that I have written has shown that increasing the frequency of SSD on OMEGA to approximately $10 \mathrm{GHz}$ as planned will not hurt the third harmonic generation conversion efficiency significantly. The increased bandwidth and increased frequency of SSD will make the laser beams that strike the target on OMEGA much smoother and more uniform than ever before. Therefore it is both safe and advisable to add a second tripler crystal to the OMEGA system and decrease the SSD time cycle to around 100 picoseconds. Since the conversion efficiency remains high up to approximately $30 \mathrm{GHz}$. more experiments on OMEGA may be carried out with even higher modulation frequencies. These modifications to the existing OMEGA laser should make target irradiation more uniform, leading to more uniform compression and hopefully, a higher energy yield.

\section{References:}

1. R. S. Craxton, R. L. McCrory, J. M. Soures, "Progress in Laser Fusion," Scientific American, vol. 255, p. 5, August, 1986.

2. R. S. Craxton, "High Efficiency Frequency Tripling Schemes for High-Power Nd:Glass Lasers", IEEE J. Quantum Electronics, vol. QE-17, No. 9, p. 1780, Sept. 1981. 
3. D. Eimerl, J. M. Auerbach, C. E. Barker, D. Milam, and P. Milonni, "Multicrystal designs for efficient third-harmonic generation", Optics Lett., Vol. 22, NO. 16, 1208-1210 (1997).

4. S. Oskoui; "Broad-Bandwidth Frequency Conversion", 1996 Summer Research Program for High School Juniors at the University of Rochester's Laboratory for Laser Energetics (1996).

5. A. Babushkin, R. S. Craxton, S. Oskoui, M. J. Guardalben, R. L. Keck, and W. Seka, "Demonstration of dual-tripler broadband and third-harmonic generation and implications for OMEGA and the NIF", to be published, Proc. Soc. Photo-Opt. Instrum. Eng., proceedings of Solid State Lasers Application to ICF, June 8-12, Monterey, CA (1998).

6. S. Skupsky, R. W. Short, T. Kessler, R. S. Craxton, S. Letzring, and J. M. Soures, "Improved laser-beam uniformity using angular dispersion of frequency-modulated light", J. Appl. Phys., Vol. 66, No. $8,3456-3462$ (1989).

7. M. Wu, "Computer Simulations of Harmonic-Frequency Conversion", 1995 Summer Research Program for High School Juniors at the University of Rochester's Laboratory for Laser Energetics (1995).

8. Rothenberg, J., "Comparison of beam-smoothing methods for direct-drive intertial confinement fusion", J. Opt. Soc. Am. B, Vol. 14, No. 7, 1664-1670 (1997).

\section{Acknowledgments:}

I would like to thank Dr. R. S. Craxton for running this year's Summer High School Program, and for allowing me the chance to work on this project. I also wish to express my gratitude to Dan Bouk and Devon Battaglia from last year's program, as well as the other students from this year's summer high school program, for assisting me at many times. I would especially like to thank Dr. Craxton again, since his help and guidance was instrumental to my completion of this project. 